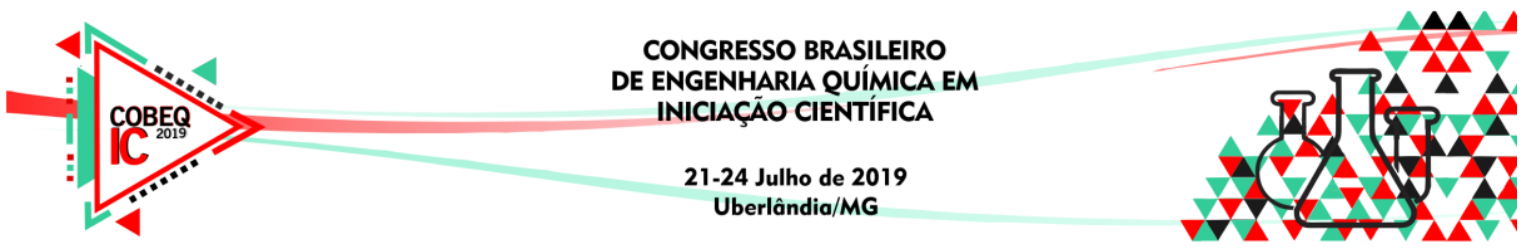

\title{
CARACTERIZAÇÃO DAS PROPRIEDADES FÍSICO- QUÍMICAS DE SOLUÇÕES AQUOSAS DE SULFATO DE SÓDIO E CARBONATO DE SÓDIO
}

\author{
G. S. PROENÇA ${ }^{1}$, V. M. V. CRUZ ${ }^{1}$, L. C. MAGALHÃES ${ }^{1}$ e J. V. H. DANGELO ${ }^{1}$ \\ ${ }^{1}$ Universidade Estadual de Campinas, Faculdade de Engenharia Química \\ E-mail para contato: g198066@ dac.unicamp.br
}

\begin{abstract}
RESUMO - O estudo realizado visa complementar a literatura, já que há poucos trabalhos com enfoque nas medições de massa específica, sólidos totais e viscosidade dinâmica de soluções aquosas de $\mathrm{Na}_{2} \mathrm{CO}_{3}$ e $\mathrm{Na}_{2} \mathrm{SO}_{4}$ sob pressão atmosférica. As temperaturas estudadas são $25{ }^{\circ} \mathrm{C}, 50{ }^{\circ} \mathrm{C}$ e $80^{\circ} \mathrm{C}$ e a variação da composição das soluções está entre água pura e próximo à saturação. Os dados foram correlacionados com o auxílio de regressão múltipla, obtendo valores em concordância com a literatura.
\end{abstract}

\section{INTRODUÇÃO}

O processo Kraft de polpação da madeira para produção de celulose e papel utiliza uma solução aquosa de sulfato e sulfeto de sódio, determinada licor branco, na etapa de cozimento da madeira. O licor negro é um subproduto desta etapa de cozimento e contém, além de lignina, sais de sódio dissolvidos, como o carbonato de sódio e o próprio sulfato de sódio. Esse licor é posteriormente processado em uma unidade de recuperação, sendo os sais recuperados e reciclados no processo. No setor de recuperação, os sais de sódio encontram-se presentes em várias correntes do processo. Cardoso et. al., (2008) afirmaram que as características físicoquímicas do licor negro são influenciadas, principalmente, pela sua composição química e condição de operação industrial, assim como as correntes derivadas desse licor. Portanto, compreender como a composição influencia algumas propriedades físico-químicas de interesse, é importante para compreender melhor o processo e propor melhorias visando maior controle e eficiência.

Os compostos inorgânicos tratados neste estudo têm propriedades que se relacionam com a concentração destes sais em meio aquoso. Segundo Gonçalves e Kestin (1981), a viscosidade relativa da solução aquosa de carbonato de sódio aumenta de acordo com a elevação da concentração, e diminui de acordo com o aquecimento da solução. Isto é o esperado devido ao forte efeito da temperatura na viscosidade, uma vez que o aumento da temperatura reduz a viscosidade dinâmica dos líquidos. Além disso, demonstraram que a massa específica da solução de carbonato de sódio tem o mesmo comportamento que a viscosidade dinâmica quando alteradas as concentrações e temperaturas.

O objetivo deste estudo é realizar medidas experimentais de viscosidade, massa específica e sólidos totais de soluções aquosas de sulfato de sódio e de carbonato de sódio, visando 


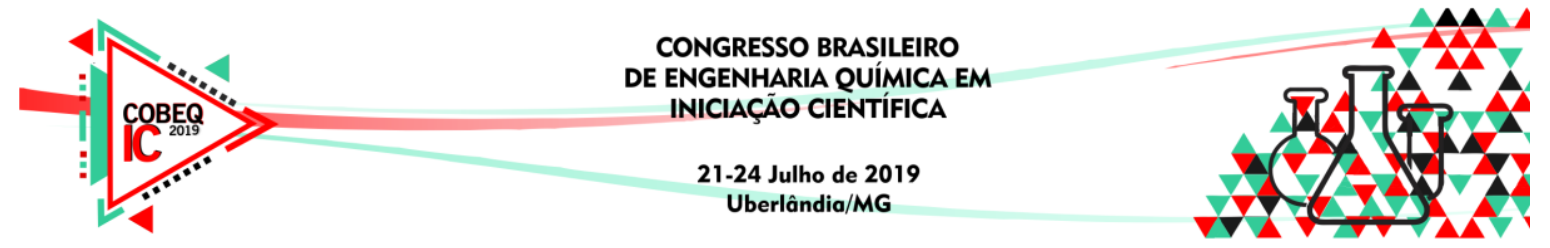

analisar o impacto destes sais sobre a água e verificar a existência de correlações entre essas propriedades e a concentração dos sais.

\section{METODOLOGIA}

As soluções de sulfato de sódio e carbonato de sódio foram preparadas utilizando um balão volumétrico $\left(250 \mathrm{~mL}\right.$ ), com concentrações de 2,$5 ; 5,0 ; 10,0 ; 12,5 ; 15,0$ e 20,0 g.L -1 $^{-1}$ (para o carbonato de sódio foram ainda preparadas soluções de 30,50 e $70 \mathrm{~g} . \mathrm{L}^{-1}$ além dessas). As soluções foram aquecidas em banho termostático a três temperaturas, $25^{\circ} \mathrm{C}, 50^{\circ} \mathrm{C}$ e $80^{\circ} \mathrm{C}$, após esse procedimento foram determinados os valores da massa específica e viscosidade para as temperaturas supracitadas, além da determinação dos sólidos totais.

Para a determinação da massa específica, foi utilizada a técnica da picnômetria utilizando picnômetros de $50 \mathrm{~mL}$, os quais foram previamente calibrados a partir da relação da massa de água que ocupa o volume do picnômetro e sua massa específica na temperatura determinada, obtida do banco de dados do NIST (National Institute of Standards and Technology https://www.nist.gov). Feito isto, realizou-se o cálculo do valor da massa específica a partir da relação da massa de solução que ocupa o volume do picnômetro real na temperatura de interesse, conforme a Equação 1.

$$
\rho_{\mathrm{Amostra}(\mathrm{T}, \mathrm{c})}{ }^{*}=\frac{m_{\mathrm{Amostra}}}{v_{\text {Real }(\mathrm{T})}}
$$

no qual, $\mathrm{m}_{\text {Amostra }}$ é a massa da amostra $(\mathrm{kg})$ obtida pela diferença entre a massa do picnômetro vazio e cheio e $\rho^{*} \operatorname{Amostra(T,c)}\left(\mathrm{kg} \cdot \mathrm{m}^{-3}\right)$ é a massa específica da amostra na temperatura de medição.

As análises de viscosidade dinâmica foram feitas a partir do viscosímetro de Ostwald. Seu funcionamento baseia-se na contagem de tempo que um fluido percorre a distância entre dois meniscos dentro de um capilar. Com este tempo, é possível calcular a viscosidade dinâmica das amostras pela Equação 2, utilizando-se do valor da massa específica da amostra, que fora previamente determinada.

$$
\eta_{\text {Amostra(T, c })}^{*}=\frac{\eta_{\text {Água(T, 0) }}^{*} \cdot \rho_{\text {Amostra(T,c) }}{ }^{*} \cdot t_{\text {Amostra(T,c) }}}{\rho_{\text {Água }(\mathrm{T}, 0)}^{*} \cdot t_{\text {Água }(\mathrm{T}, 0)}}
$$

no qual, $\eta^{*}$ Água(T,0) é a viscosidade dinâmica (cP) da água na temperatura de referência obtida no NIST, $\mathrm{t}_{\mathrm{Amostra}(\mathrm{T}, \mathrm{c})}$ é o tempo (s) decorrido para a amostra percorrer a distância entre os dois meniscos do capilar, t́́gua(T,0) é o tempo (s) decorrido para a água percorrer a distância entre os dois meniscos do capilar e $\eta^{*}$ Amostra(T,c) é a viscosidade dinâmica (cP) da amostra na temperatura de medição. Para a análise dos dados de massa específica e viscosidade dinâmica, foi feito o uso de propriedade relativas. As mesmas podem ser calculadas pelas Equação 3.

$$
G_{\text {Relativa }(T, C)}^{*}=\frac{G^{*} \operatorname{Amostra}(T, C)}{G_{\text {Água }(T, 0)}}
$$

no qual, $\mathrm{G}$ Relativa $(\mathrm{T}, \mathrm{c})^{*}$ é a propriedade relativa da amostra a ser calculada na temperatura desejada, $\mathrm{G}$ Amostra $(\mathrm{T}, \mathrm{c})^{*}$ é o valor empírico da propriedade e $\mathrm{G}$ Água $(\mathrm{T}, 0)^{*}$ é o valor da propriedade 


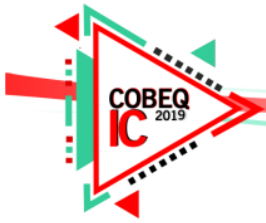

da água na temperatura inicial de referência. Os valores obtidos do NIST e utilizados como referência são apresentados na Tabela 1 .

Tabela 1 - Valores Utilizados para o Cálculo das Propriedades Relativas

\begin{tabular}{|c|c|c|c|}
\hline \multirow{2}{*}{ Propriedade } & \multicolumn{3}{|c|}{ Temperatura $\left({ }^{\circ} \mathrm{C}\right)$} \\
\cline { 2 - 4 } & 25 & 50 & 80 \\
\hline \hline Massa específica $\left(\mathrm{kg} \cdot \mathrm{m}^{-3}\right)$ & 997,05 & 988,03 & 971,78 \\
\hline Viscosidade Dinâmica (cP) & 0,8899 & 0,5468 & 0,3543 \\
\hline
\end{tabular}

Fonte: National Institute of Standards and Technology

(https://nvlpubs.nist.gov/nistpubs/Legacy/IR/nistir5078.pdf)

\section{RESULTADOS}

A Tabela 2 contêm os dados de massa específica relativa e viscosidade dinâmica relativa das concentrações da solução de sulfato de sódio. A Tabela 3 fornece os valores de massa específica relativa e viscosidade dinâmica relativa das concentrações da solução de carbonato de sódio. As colunas $\rho^{*}$ Relativa e $\eta^{*}$ Relativa, representam, respectivamente a massa específica relativa e a viscosidade dinâmica relativa da solução, determinadas a uma dada temperatura, calculadas pela Equação 3.

Tabela 2 - Relação das Propriedades Relativas Estudadas da Solução de Sulfato de Sódio

\begin{tabular}{|c|c|c|c|c|}
\hline Número da Solução & Concentração $\left(\mathrm{kg} / \mathrm{m}^{3}\right)$ & Temperatura $\left({ }^{\circ} \mathrm{C}\right)$ & $\rho *_{\text {Relativa }}$ & $\eta^{*}$ Relativa \\
\hline \multirow{3}{*}{$1 \mathrm{~S}$} & \multirow{3}{*}{2,5} & 25 & 1,0022 & 1,0238 \\
\hline & & 50 & 0,9946 & 1,0152 \\
\hline & & 80 & 0,9988 & 0,9204 \\
\hline \multirow{3}{*}{$2 S$} & \multirow{3}{*}{5,0} & 25 & 1,0066 & 1,0306 \\
\hline & & 50 & 0,9979 & 1,0422 \\
\hline & & 80 & 1,0022 & 0,9266 \\
\hline \multirow{3}{*}{$3 \mathrm{~S}$} & \multirow{3}{*}{10,0} & 25 & 1,0079 & 1,0534 \\
\hline & & 50 & 1,0031 & 1,0812 \\
\hline & & 80 & 1,0059 & 0,9389 \\
\hline \multirow{3}{*}{$4 S$} & \multirow{3}{*}{12,5} & 25 & 1,0122 & 1,0792 \\
\hline & & 50 & 1,0082 & 1,0842 \\
\hline & & 80 & 1,0083 & 0,9423 \\
\hline \multirow{3}{*}{$5 \mathrm{~S}$} & \multirow{3}{*}{15,0} & 25 & 1,0132 & 1,0937 \\
\hline & & 50 & 1,0101 & 1,1080 \\
\hline & & 80 & 1,0089 & 0,9506 \\
\hline \multirow{3}{*}{$6 \mathrm{~S}$} & \multirow{3}{*}{20,0} & 25 & 1,0192 & 1,1074 \\
\hline & & 50 & 1,0170 & 1,1338 \\
\hline & & 80 & 1,0173 & 0,9804 \\
\hline
\end{tabular}




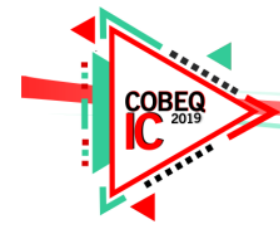

Tabela 3 - Relação das Propriedades Relativas Estudadas da Solução de Carbonato de Sódio

\begin{tabular}{|c|c|c|c|c|}
\hline Número da Solução & Concentração $\left(\mathrm{kg} / \mathrm{m}^{3}\right)$ & Temperatura $\left({ }^{\circ} \mathrm{C}\right)$ & $\rho *_{\text {Relativa }}$ & $\eta{ }^{*}$ Relativa \\
\hline \multirow{3}{*}{$1 \mathrm{C}$} & \multirow{3}{*}{2,5} & 25 & 1,0013 & 1,0766 \\
\hline & & 50 & 1,0060 & 0,7104 \\
\hline & & 80 & 1,0036 & 0,8782 \\
\hline \multirow{3}{*}{$2 \mathrm{C}$} & \multirow{3}{*}{5,0} & 25 & 1,0059 & 1,0579 \\
\hline & & 50 & 1,0072 & 0,7130 \\
\hline & & 80 & 1,0059 & 0,8786 \\
\hline \multirow{3}{*}{$3 \mathrm{C}$} & \multirow{3}{*}{10,0} & 25 & 1,0090 & 1,0637 \\
\hline & & 50 & 1,0084 & 0,7363 \\
\hline & & 80 & 1,0099 & 0,9825 \\
\hline \multirow{3}{*}{$4 \mathrm{C}$} & \multirow{3}{*}{12,5} & 25 & 1,0117 & 1,1177 \\
\hline & & 50 & 1,0117 & 0,7827 \\
\hline & & 80 & 1,0105 & 0,9919 \\
\hline \multirow{3}{*}{$5 \mathrm{C}$} & \multirow{3}{*}{15,0} & 25 & 1,0144 & 1,1069 \\
\hline & & 50 & 1,0125 & 0,8047 \\
\hline & & 80 & 1,0122 & 1,0056 \\
\hline \multirow{3}{*}{$6 \mathrm{C}$} & \multirow{3}{*}{20,0} & 25 & 1,0191 & 1,1675 \\
\hline & & 50 & 1,0150 & 0,8815 \\
\hline & & 80 & 1,0134 & 1,0087 \\
\hline \multirow{3}{*}{$7 \mathrm{C}$} & \multirow{3}{*}{25,0} & 25 & 1,0235 & 1,1521 \\
\hline & & 50 & 1,0166 & 0,8868 \\
\hline & & 80 & 1,0182 & 1,0270 \\
\hline \multirow{3}{*}{$8 \mathrm{C}$} & \multirow{3}{*}{30,0} & 25 & 1,0282 & 1,2087 \\
\hline & & 50 & 1,0207 & 1,1145 \\
\hline & & 80 & 1,0200 & 1,0317 \\
\hline \multirow{3}{*}{$9 \mathrm{C}$} & \multirow{3}{*}{50,0} & 25 & 1,0448 & 1,3116 \\
\hline & & 50 & 1,0441 & 1,1459 \\
\hline & & 80 & 1,0382 & 1,0601 \\
\hline \multirow{3}{*}{$10 \mathrm{C}$} & \multirow{3}{*}{70,0} & 25 & 1,0748 & 1,4572 \\
\hline & & 50 & 1,0681 & 1,2457 \\
\hline & & 80 & 1,0636 & 1,1542 \\
\hline
\end{tabular}

Ao observar as Tabelas 2 e 3, nota-se que as propriedades relativas têm um comportamento linear quando analisadas sob uma mesma temperatura, em que os valores das propriedades aumentam conforme se aumenta a concentração da solução. Além disso, percebese e que os valores de uma propriedade a uma dada concentração diminuem conforme aumentase a temperatura. As conclusões obtidas, quando comparadas com a literatura demonstrada por Gonçalves e Kestin (1981), estão em concordância, confirmando a confiabilidade dos resultados.

\subsection{Correlações}

As correlações para predizer o comportamento dos sais em soluções aquosas foram feitas por meio do software Minitab ${ }^{\circledR}$ v.8. A regressão múltipla modela a relação entre a propriedade relativa, a temperatura e a concentração da solução. A Equação 4 representa este ajuste, em que se obteve, no mínimo, $90 \%$ da variação da propriedade relativa sendo explicada por tal modelo de regressão, quando estudado todos os casos esta análise se propõe a fazer. 


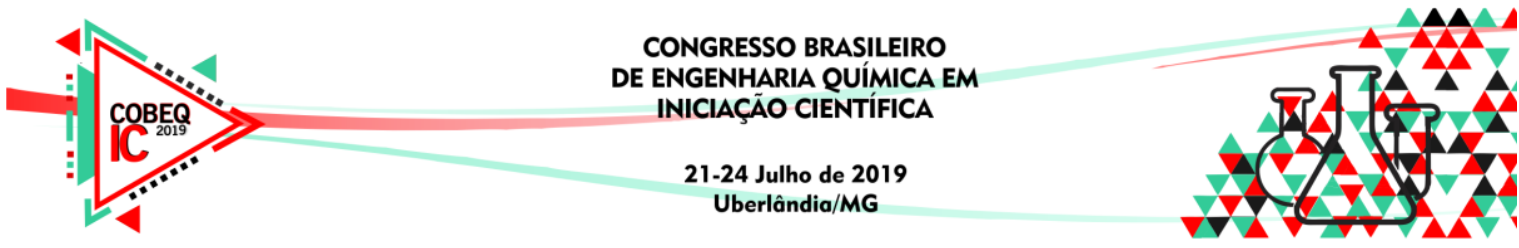

$$
G_{\text {Relativa }(T, c)}{ }^{*}=\mathrm{A}+\mathrm{B} \cdot T+C \cdot c+D \cdot T^{2}+E \cdot c^{2}+F \cdot T \cdot C
$$

no qual, $T$ é a temperatura $\left({ }^{\circ} \mathrm{C}\right)$ em que se quer analisar a propriedade, $c$ é a concentração (g.L $\mathrm{L}^{-1}$ ) da solução de sulfato de sódio ou de carbonato de sódio, dependendo do enfoque do estudo, e A, B, C, D, E e F são os coeficientes das soluções de sulfato de sódio e carbonato de sódio fornecidos pela Tabela 4.

Tabela 4 - Coeficientes das Propriedades Relativas das Soluções de $\mathrm{Na}_{2} \mathrm{SO}_{4}$ e $\mathrm{Na}_{2} \mathrm{CO}_{3}$

\begin{tabular}{|c|c|c|c|c|c|c|}
\hline \multirow{2}{*}{ Propriedade } & \multicolumn{7}{|c|}{ Coeficientes Sulfato de Sódio } \\
\cline { 2 - 7 } & A & B & C & D & E & F \\
\hline$\rho^{*}$ Relativa & 1,518 & $-0,00316$ & 0,001049 & 0,000005 & 0 & 0 \\
\hline$\eta^{*}$ Relativa & $-8,011$ & 0,05724 & 0,01784 & $-0,000091$ & 0 & $-0,00004$ \\
\hline \multirow{2}{*}{ Propriedade } & \multicolumn{7}{|c|}{ Coeficientes do Carbonato de Sódio } \\
\cline { 2 - 7 } & A & B & C & D & E & F \\
\hline$\rho^{*}$ Relativa & 0,99978 & 0,000012 & 0,001534 & 0 & 0,000007 & $-0,000003$ \\
\hline$\eta^{*}$ Relativa & 28,6 & $-0,1691$ & 0,02175 & 0,000257 & 0 & $-0,000048$ \\
\hline
\end{tabular}

Deste modo, a Equação 4 e os coeficientes presentes na Tabela 4 podem ser utilizados para predizer as propriedades relativas para valores específicos da temperatura e da concentração das soluções, ou para encontrar as configurações para a temperatura e concentração que correspondem a um valor desejado ou a uma amplitude de valores para as propriedades relativas. A análise estatística dos valores preditos e medidos, ou seja, a distribuição dos resíduos gerados pelo modelo, estão presentes nas Figuras 1 e 2.

Figura 1 - Resíduos do modelo obtido para viscosidade (a) e massa específica (b) do $\mathrm{Na}_{2} \mathrm{SO}_{4}$.
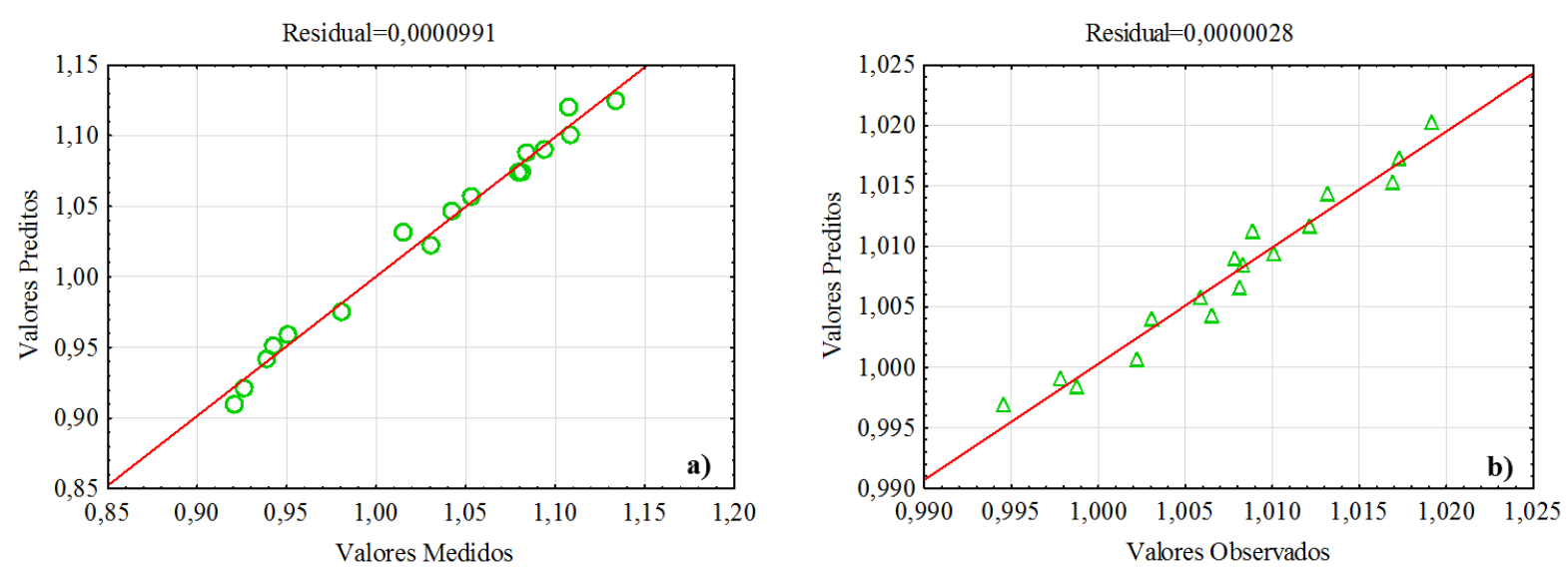

Para validar o modelo obtido foram preparadas mais duas soluções de diferentes concentrações, sendo realizadas as medições da massa específica e viscosidade em outra temperatura que não as medidas. Os dados experimentais e preditos, bem como os parâmetros utilizados nas soluções estão presentes na Tabela 5. 


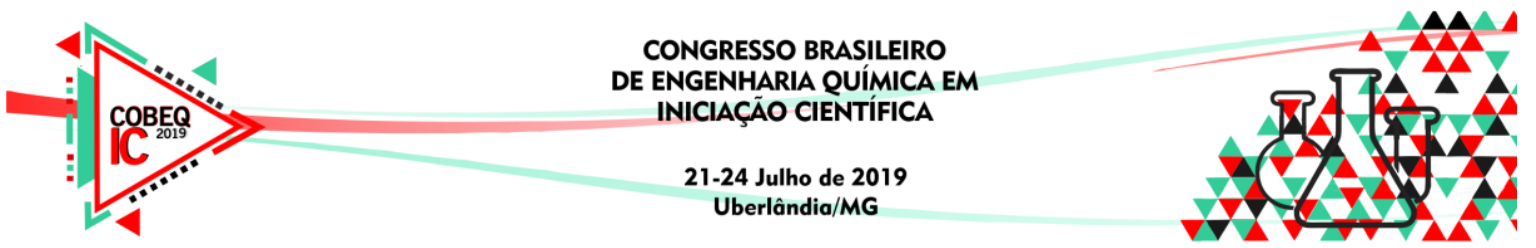

Figura 2 - Resíduos do modelo obtido para viscosidade (a) e massa específica (b) do $\mathrm{Na}_{2} \mathrm{CO}_{3}$.
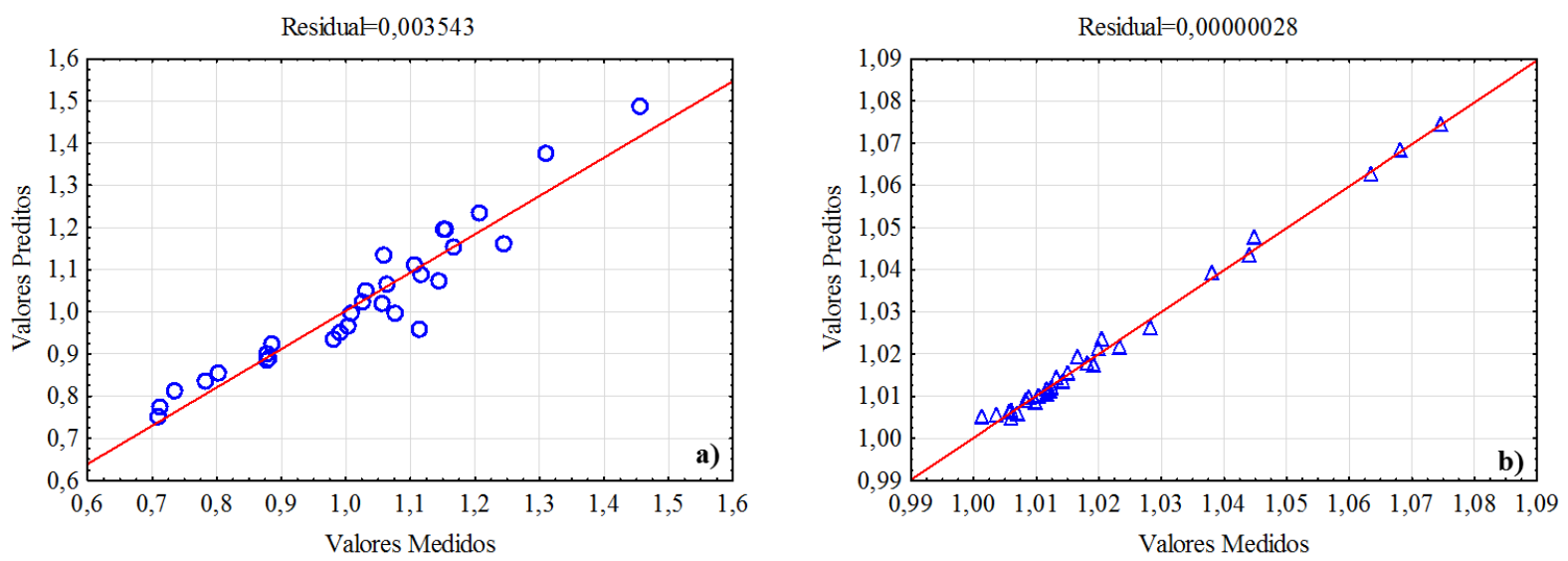

Tabela 5 - Dados da validação do modelo estatístico obtido de $\mathrm{Na}_{2} \mathrm{SO}_{4}$ e $\mathrm{Na}_{2} \mathrm{CO}_{3}$

\begin{tabular}{|c|c|c|c|c|c|c|c|}
\hline \multicolumn{2}{|l|}{ Parâmetros } & $\rho_{\text {Exp. }}\left(\mathrm{kg} \cdot \mathrm{m}^{-3}\right)$ & $\rho_{\text {Prevista }}\left(\mathrm{kg} \cdot \mathrm{m}^{-3}\right)$ & $\begin{array}{c}\text { Desvi } \\
0\end{array}$ & $\eta_{\text {Exp. }}(\mathrm{cP})$ & $\eta_{\text {Prevista }}(\mathrm{cP})$ & $\begin{array}{c}\text { Desvi } \\
\mathrm{o}\end{array}$ \\
\hline Temperatura & $40^{\circ} \mathrm{C}$ & \multirow{2}{*}{1,05} & \multirow{2}{*}{1,0385} & \multirow{2}{*}{$0,7 \%$} & \multirow{2}{*}{1,0955} & \multirow{2}{*}{1,1173} & \multirow{2}{*}{$2,0 \%$} \\
\hline Concentração $\mathrm{Na}_{2} \mathrm{CO}_{3}$ & 40 g.L. ${ }^{-1}$ & & & & & & \\
\hline Temperatura & $40^{\circ} \mathrm{C}$ & \multirow{2}{*}{1,05} & \multirow{2}{*}{1,0272} & \multirow{2}{*}{$2,3 \%$} & \multirow{2}{*}{1,0115} & \multirow{2}{*}{1,0325} & \multirow{2}{*}{$2,1 \%$} \\
\hline Concentração $\mathrm{Na}_{2} \mathrm{SO}_{4}$ & 8 g. $\mathrm{L}^{-1}$ & & & & & & \\
\hline
\end{tabular}

Com a validação do modelo é possível não só predizer o comportamento das propriedades com a sua composição e temperatura, mas também utilizar esses dados para entender como os sais em solução aquosa afetam intensivamente o valor das mesmas, podendo ser aplicadas em diversos processos industriais, entre eles o de celulose e papel.

\section{CONCLUSÃO}

As correlações para massa específica relativa e viscosidade dinâmica relativa em soluções de sulfato de sódio e carbonato de sódio, em função da temperatura e concentração desses sais, foram consideradas satisfatórias por apresentar resultados compatíveis com os valores experimentais e dados relatados na literatura.

\section{REFERÊNCIAS}

CARDOSO, M.; PASSOS, M. L.; OLIVEIRA, E. D. de. Licor negro de eucalipto Kraft proveniente de indústrias brasileiras: características químicas, físicas e seu processamento na unidade de recuperação. O Papel, São Paulo, v. 67, n. 2, p. 57-83, mai. 2008.

GONÇALVES, F. A.; KESTIN, J. The Viscosity of $\mathrm{Na}_{2} \mathrm{CO}_{3}$ and $\mathrm{K}_{2} \mathrm{CO}_{3}$ Aqueous Solutions in the Range $20-60^{\circ} \mathrm{C} .1981$.

NIST - National Institute of Standards and Technology. Disponível em: < https://webbook.nist.gov/cgi/cbook.cgi?Name=benzene\&Units=SI >. Acesso em 09 de abril de 2019. 